\title{
Ruptura espontánea bilateral del tendón extensor largo del pulgar
}

\section{Bilateral spontaneous rupture of the extensor pollicis longus}

\author{
Roberto Pablo Pareyón Valero, ${ }^{\star}$ Ranulfo Romo Rodríguez ${ }^{\ddagger}$
}

\section{RESUMEN}

La ruptura bilateral del tendón extensor largo de pulgar es poco común y mucho más si se presenta de manera esporádica y espontánea. Hay pocos reportes en la literatura acerca de esta lesión; la mayoría de éstos tiene alguna razón anatómica que puede explicar este evento. En este caso se presenta una paciente con múltiples falanges hipoplásicas que sufre de ruptura bilateral espontánea del tendón extensor largo del pulgar; asimismo, se presenta el abordaje diagnóstico y manejo terapéutico, al igual que la probable etiología.

Palabras clave: Extensor largo del pulgar, bilateral, espontánea, transferencia.

Nivel de evidencia: IV

\begin{abstract}
Bilateral rupture of the extensor pollicis longus tendon is uncommon and much more if it occurs sporadically and spontaneously. There are few case reports in the medical literature about this pathology, with most of these cases having somo anatomical reason that can explain this event. In this case, a patient with multiple hypoplasic phalanges who suffers a bilateral spontaneous rupture of the extensor pollis longus tendon is presented, as well as the diagnostic approach and therapeutic management as well as the probable etiology.
\end{abstract}

Keywords: Extensor pollicis longus, bilateral, spontaneous, transference.

Level of evidence: $I V$
* Residente de segundo año de Traumatología y Ortopedia.

‡ Cirujano de extremidad superior y microcirugía.

Centro Médico ABC

Recibido para publicación: 31/01/2020. Aceptado: 02/03/2020.

\section{Correspondencia:}

Dr. Roberto Pablo Pareyón Valero

Calle Sur 132 Núm. 122, Col. Las Américas, 01120,

Álvaro Obregón, Ciudad de México.

Tel: 55-3555-5544

E-mail: ropareyon@gmail.com

\section{Abreviaturas:}

$E P L=$ Extensor largo del pulgar.

$\mathrm{EPI}=$ Extensor propio del índice.

USG $=$ Ultrasonido.

$\mathrm{ECRL}=$ Extensor radial largo del carpo.

\section{INTRODUCCIÓN}

El primer reporte en la literatura de rotura espontánea del tendón extensor largo del pulgar (EPL) fue escrito por Duplay en $1876 .^{1}$

Desde ese tiempo se han reportado múltiples casos de ruptura de EPL y se ha tratado de describir causas, fisiopatología y tratamiento.

Sin embargo, la ruptura bilateral del EPL es poco frecuente y se ha tratado de relacionar a enfermedades sistémicas. Las más comunes son artritis reumatoide o secundario a algún tipo de traumatismo, como una fractura de radio distal.

También se puede relacionar a inyecciones locales con esteroide, movimientos anormales o excesivos de la articulación radio-carpal, espondilitis anquilosante, infiltración de gota tofácea, mala colocación de fijadores externos en manejo de fractura, fracturas de escafoides, metástasis a radio distal, pseudoartrosis de 
escafoides, subluxación dorsal del cúbito secundario a traumatismo, deformidad de Madelung o rizartrosis. ${ }^{2}$

Este caso es el reporte de ruptura cerrada bilateral del tendón extensor largo del pulgar.

\section{CASO CLÍNICO}

Paciente femenino de 67 años de edad, diestra. Es referida por presentar dolor intenso en el dorso de la mano izquierda acompañado de edema y limitación de la movilidad, sobre todo para la extensión del pulgar después de cargar una bolsa de aproximadamente $1 \mathrm{~kg}$ de peso con la mano.

En la exploración física del pulgar izquierdo se encuentra con $50^{\circ}$ de flexión, incapacidad para la extensión de la articulación metacarpofalángica e interfalángica y para retropulsión del pulgar. La prueba de tenodesis con flexión de muñeca es positiva para ruptura de extensor largo del pulgar.

No existen antecedentes de infiltraciones o fracturas en ninguna de las manos.

Además, en ambas manos se encuentra dedo medio más corto. Clínicamente, dicho acortamiento se palpa a expensas de la falange media, la cual se ve afectada en todos los dedos restantes, menos en el dedo anular, el cual se palpa de tamaño normal.

En la radiografía de la mano izquierda (Figura 1) se encuentra una rizartrosis grado III y una braquifa-

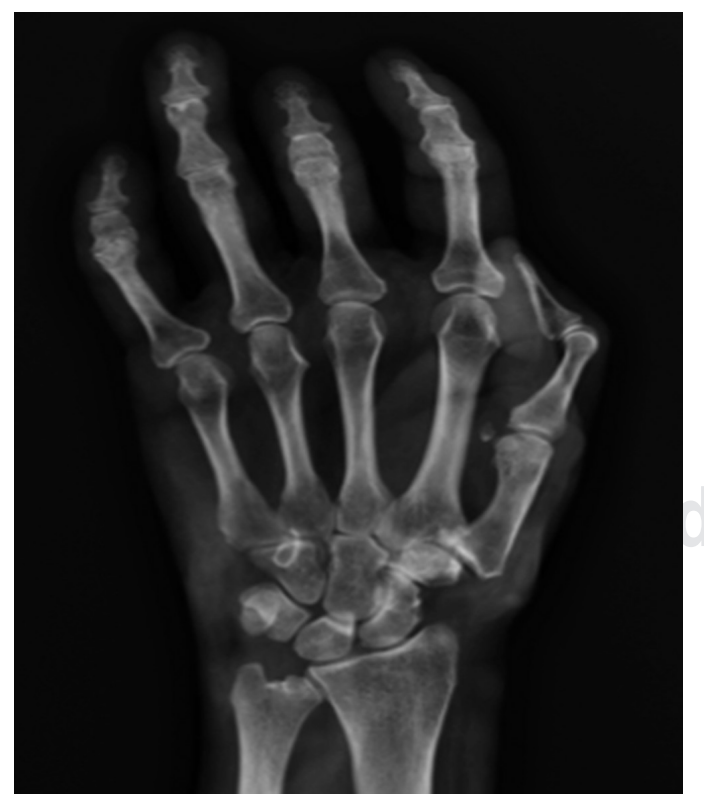

Figura 1: Radiografía anteroposterior de la mano izquierda, se observa la falange media del segundo, tercer y quinto dedo con hipoplasia. Importante rizartrosis.

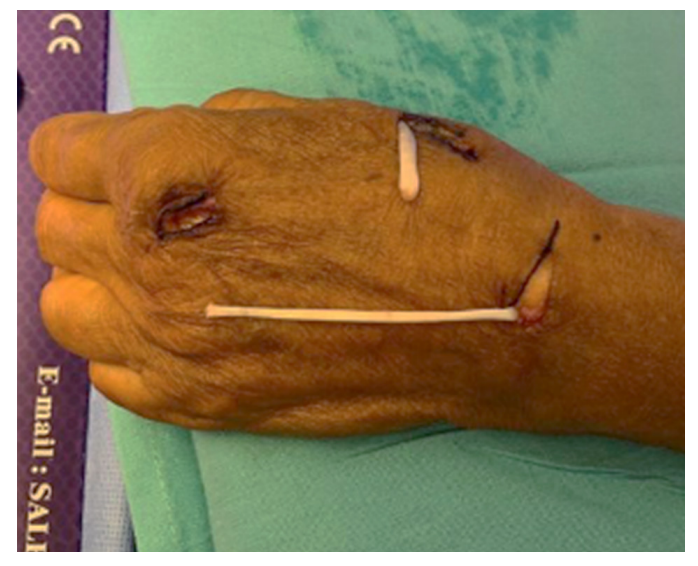

Figura 2: Aislamiento del tendón extensor largo del pulgar y la obtención del tendón extensor propio del índice previo a la transferencia en la mano izquierda.

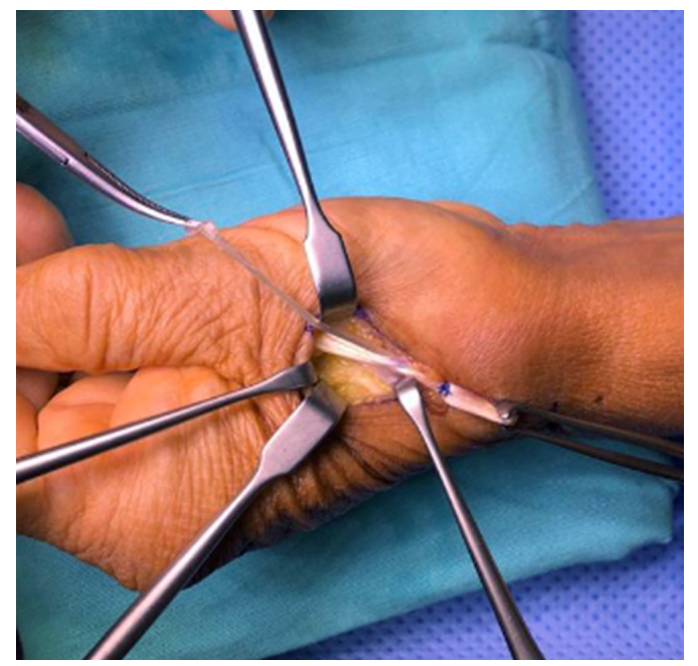

Figura 3: Transferencia tendinosa del tendón extensor largo del pulgar y del tendón propio del índice con técnica Pulvertaft en la mano izquierda.

langia del segundo al quinto dedo de la falange media, con el dedo anular con la falange media más grande de los dedos mencionados. Asimismo, un braquimetacarpo del pulgar.

Se realizó un ultrasonido (USG) por la sospecha clínica de rotura de EPL. Éste fue negativo para dicho diagnóstico y sólo reportó tenosinovitis importante del tercer compartimento extensor.

Debido al reporte negativo para rotura del EPL del USG, el manejo inicial fue conservador con terapia física. Al no haber una mejoría es referida con nosotros.

Se planea manejo quirúrgico de la mano; se realiza transferencia del tendón extensor propio del índice al extensor largo del pulgar con técnica Pulvertaft 
con sutura no absorbible pseudomonofilar de poliamida 4-0 (Supramid ${ }^{\circledR}$ ) (Figuras 2 y 3 ). Se cierran heridas con Nylon 4-0 y se protege reparación con férula antebraquipalmar hasta la articulación interfalángica del pulgar y vendaje compresivo durante 10 días. Después, se inician ejercicios de tenodesis, ejercicios pasivos de flexo-extensión a las dos semanas y ejercicios activos a las cuatro semanas, se mantuvo una protección con muñequera con Spica de pulgar entre sesiones de ejercicios.

Aproximadamente, tres meses después de la lesión inicial, al intentar abrir una puerta, tuvo sensación de chasquido en la mano derecha en región dorsal de la articulación metacarpofalángica del primer dedo, presentó dolor inmediato, edema e incapacidad al movimiento, específicamente a la extensión del pulgar.

A la exploración física del pulgar derecho presenta $60^{\circ}$ de flexión e incapacidad a la extensión interfalángica y retropulsión. La prueba de tenodesis es positiva de nuevo.

En la radiografía de la mano derecha (Figura 4) se encuentra el mismo hallazgo de braquifalangia del segundo al cuarto dedo, una vez más a expensas de la falange media con el dedo anular con la falange más grande y una rizartrosis grado II.

Debido al reporte negativo del ultrasonido de la mano contralateral, así como la clínica congruente con rotura del tendón extensor largo del pulgar, no se realizó USG de la mano derecha.

Se realizó el mismo procedimiento y protocolo de rehabilitación en la mano derecha.

\section{DISCUSIÓN}

Una ruptura espontánea de tendón se define como una escisión que se presenta durante actividades que usualmente no dañan o lesionan estructuras musculotendinosas.

Son mucho más comunes las rupturas unilaterales espontáneas; sin embargo, se han reportado en la literatura rupturas bilaterales sin motivo específico.

Las causas de la ruptura cerrada y espontánea del EPL se dividen en dos grandes grupos: la teoría vascular y la teoría mecánica.

La teoría vascular fue propuesta y fundamentada por Engkvist y colaboradores, ${ }^{3}$ en la que se realizó un estudio microangiopático con pacientes postfracturados de radio distal, se encontró que muy cerca del tubérculo de Lister el EPL tiene una mala vascularidad, ya que la nutrición del tendón en esta zona depende de la difusión. Propone que la hemorragia producida por cualquier causa cerca de esta zona limita la difusión y secundariamente la nutrición del tendón.

Por otro lado, la teoría mecánica toma fuerza con el artículo publicado por Mannerfelt y su equipo, ${ }^{4}$ quienes describieron la ruptura del EPL en pacientes con fracturas de radio distal simples metafisarias con desviación dorsal (Colles) y artritis reumatoide.

Incluso hablando de artritis reumatoide, $\mathrm{Ryu}^{5}$ menciona que son múltiples las causas por las cuales esta patología puede ocasionar rupturas de tendones extensores, tales como invasión de granulaciones reumáticas en el tendón, subluxación dorsal del cúbito, alteraciones vasculares por aumento de la sinovitis y granulaciones múltiples, así como uso prolongado de esteroides. En su artículo recomienda la tenosinovectomía profiláctica.

Denman ${ }^{6}$ propone una teoría de ruptura en momentos de hiperextensión forzada de la muñeca, en el que el tendón puede quedar atrapado y lesionarse entre el tubérculo de Lister y la base del tercer metacarpiano.

Algunos autores mencionan otras causas además de las mencionadas previamente, incluyendo también deportes como el kick boxing. ${ }^{7}$

Sólo se han reportado cinco casos de ruptura bilateral del tendón extensor largo del pulgar, todos con

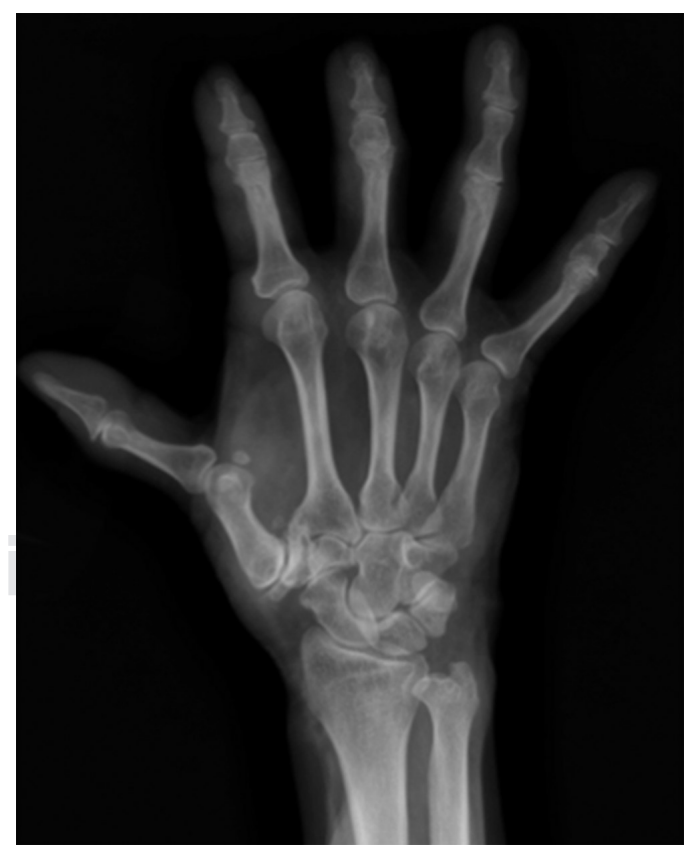

Figura 4: Radiografía anteroposterior de la mano derecha, se observan falanges medias hipoplásicas, así como primer metacarpiano hipoplásico. Importante rizartrosis. 
An Med (Mex) 2020; 65 (1): 67-71

condiciones asociadas. Dos de ellos por evento traumático ${ }^{2,8}$ y otros dos después de recibir infiltración con esteroides. ${ }^{9,10}$ Mientras que uno más reportado por Galluci ${ }^{11}$ fue secundario a una anormalidad anatómica en el tubérculo de Lister.

Es importante mencionar las braquifalangias de las falanges medias descritas previamente que tiene esta paciente; en realidad, el término correcto debe ser «falange hipoplásica», ya que es una deficiencia simétrica sin una deformidad asociada.

La hipoplasia de toda la mano es el $0.8 \%$ de todas las deformidades y la braquidactilia es el 5.2\%, la más frecuente es la falange media, la cual es la última parte del esqueleto digital en osificarse, como en nuestro paciente.

De hecho, la braquidactilia ha tenido un impacto fuerte en la literatura genética como ejemplo de la herencia mendeliana, siendo ésta autosómica dominante. También se han reportado casos aislados sin etiología verdaderamente clara.

Se relaciona también con malformaciones en los dedos de los pies o síndromes como Treacher Collins, Bloom, Cornelia de Lange, Holt-Oram, Silver y Poland.

En nuestro paciente no se logra definir un síndrome como tal, lo cual es lo más frecuente. Existe una clasificación hecha por Bell en 1951, la cual ha sido modificada y describe las deformaciones con sus asociaciones; esta paciente encaja más en el grupo de una braquidactilia tipo A. ${ }^{12}$

Recientemente, se describió una clasificación de anomalías congénitas de miembro superior llamada Oberg, Manske, Tonkin (OMT), la cual pretende dar una explicación genética y una clasificación etiológica más completa que la de Swanson o Bell y, de esta manera, generar una comunicación global e interdisciplinaria. ${ }^{13}$

La paciente no tenía ninguna de las condiciones etiológicas antes mencionadas; no obstante, es importante mencionar el antecedente de las deformidades en las falanges, así como la rizartrosis importante que presenta en ambas manos, la cual es la etiología más probable de ambas rupturas.

Es por esto que pensamos que la causa real de la rotura bilateral es la rizartrosis que presenta la paciente con formación de osteofitos, así como el hecho de presentar braquifalangias y braquimetacarpos sugiere la posibilidad de que exista también una deformidad en el mismo tendón.

En diferentes estudios se ha tratado de encontrar la sensibilidad y especificidad del ultrasonido como método diagnóstico, descubriendo grandes varia- ciones según el estudio. Esto es normal, sobre todo porque es operador dependiente, además de que no en todas las series se comprobó con cirugía o algún otro estudio de imagen. Sin embargo, Lee ${ }^{14}$ encontró ciertas características en el tendón extensor largo del pulgar como discontinuidad, pseudomasa, disminución en la ecogenicidad del tendón, retracción del tendón y colección de líquido en la vaina del mismo. El hallazgo más específico es la discontinuidad (100\%), seguida de la pseudomasa $(73 \%)$ a diferencia de otros tendones extensores.

En otro estudio, Santiago ${ }^{15}$ cuenta con una serie de 12 casos de ruptura del EPL, en los cuales se realizó ultrasonido en comparación con resonancia magnética, en el cual se encontró una sensibilidad y especificidad arriba de $80 \%$ en rupturas severas o completas. No reporta rupturas parciales y recomienda el uso del ultrasonido siempre y cuando exista experiencia.

A pesar de lo anterior, en nuestro caso hubo un falso negativo al utilizar ultrasonido, por lo que la clínica de nuestro paciente con la incapacidad para generar la movilidad del dedo pulgar tanto para extensión como para retropulsión, sumado a la prueba de tenodesis positiva, nos habla de que la clínica prevalece sobre el ultrasonido para la toma de decisiones en el manejo de pacientes con estas lesiones.

Las opciones de tratamiento que se tienen son la reparación término-terminal, injerto de tendón o transferencia tendinosa. La elección de varios autores es la transferencia tendinosa, sobre todo por la menor formación de tejido fibroso en la reconstrucción y menor resultado de contracturas, teniendo la opción de hacerlo con el extensor propio del índice (EPI) o, incluso, con el extensor radial largo del carpo (ECRL); dentro de estas dos opciones se elige la del EPI por la fácil obtención a la altura de las articulaciones metacarpofalángicas y no se pierde función del índice en lo absoluto. ${ }^{16}$

\section{CONCLUSIONES}

Es un caso que puede servir de ayuda en la práctica, no sólo de cirujanos de mano, sino también de ortopedistas al tener presente que la ruptura del tendón extensor largo del pulgar puede tener múltiples causas y muchas de ellas, inclusive, pueden debutar con esta patología, razón por la cual vale la pena comenzar con diferentes abordajes diagnósticos. Además, es importante tener en mente un algoritmo de tratamiento para estos pacientes, como el manejo conservador, encontrar pacientes candidatos para la te- 


\section{nosinovectomía y quiénes deben ser sometidos a una transferencia tendinosa, como nuestro caso.}

\section{BIBLIOGRAFÍA}

1. Duplay S. Rupture sous-cutanée du tendon du long extenseur du pouce de la main droite, au niveau de la tabatière anatomique. Flexion permanente du pouce. Rétablissement de la faculté d'extension par une opération (suture de l'extrémité de tendon rompu avec le primer radial externe). Bull Mem Soc Chir Paris. 1876; 2: 788-791.

2. Björkman A, Jörgsholm P. Rupture of the extensor pollicis longus tendon: a study of aetiological factors. Scand J Plast Reconstr Surg Hand Surg. 2004; 38 (1): 32-35.

3. Engkvist O, Lundborg G. Rupture of the extensor pollicis longus tendon after fracture of the lower end of the radius--a clinical and microangiographic study. Hand. 1979; 11: 76-86.

4. Mannerfelt L, Oetker R, Ostlund B, Elbert B. Rupture of the extensor pollicis longus tendon after Colles fracture and by rheumatoid arthritis. J Hand Surg Br. 1990; 15: 49-50.

5. Ryu J, Saito S, Honda T, Yamamoto K. Risk factors and prophylactic tenosynovectomy for extensor tendon ruptures in the rheumatoid hand. J Hand Surg Br. 1998; 23: 658-661.

6. Denman EE. Rupture of the extensor pollicis longus--a crush injury. Hand. 1979; 11: 295-298.

7. Lloyd TW, Tyler MP, Roberts AH. Spontaneous rupture of extensor pollicis longus tendon in a kick boxer. Br J Sports Med. 1998; 32: 178-179.
8. Haher JN, Haher TR, Devlin VJ, Armenti V. Bilateral rupture of extensor pollicis longus. A case report. Orthopedics. 1987; 10: $1577-1580$.

9. Anwar I, Owers KL, Eckersley R. Spontaneous rupture of the extensor pollicis longus tendon. J Hand Surg Br. 2006; 31: 457-458.

10. Payne AJ, Harris NJ, Kehoe NJ. Bilateral delayed extensor pollicis longus rupture following bilateral undisplaced distal radial fractures. Orthopedics. 2000; 23: 163.

11. Gallucci GL, Pacher N, Boretto JG, De Carli P. Bilateral rupture of the extensor pollicis longus tendon. A case report. Chir Main. 2013; 32 (3): 186-188.

12. McKusick VA. Mendelian inheritance in man: a catolog of human genes and genetic disorders. Baltimore, MD: Johns Hopkins University Press; 1994.

13. Oberg KC. Classification of congenital upper limb anomalies: towards improved communication, diagnosis, and discovery. J Hand Surg Eur Vol. 2019; 44 (1): 4-14.

14. Lee SM, Ha DH, Han SH. Differential sonographic features of the extensor pollicis longus tendon rupture and other finger tendons rupture in the setting of hand and wrist trauma. PLoS One. 2018; 13 (10): e0205111.

15. Santiago FR, Plazas PG, Fernández JM. Sonography findings in tears of the extensor pollicis longus tendon and correlation with CT, MRI and surgical findings. Eur J Radiol. 2008; 66 (1): 112-116.

16. Green DP, Pederson WC, Hotchkiss RN, Wolfe SW. Green's cirugía de la mano. 5a ed. 2 vols. New York: MARBÁN; 2017. p. 265. 